\title{
Diversidade e uso de plantas em quintais do Bairro Nossa Senhora Aparecida em Cuiabá, Mato Grosso
}

\author{
Antonio Nobre da SILVA ${ }^{*}$, Maria de Fatima Barbosa COELHO², \\ Elisangela Clarete CAMILI ${ }^{2}$
}

\author{
${ }^{1}$ Instituto Federal de Educação, Ciência e Tecnologia de Mato Grosso, Cáceres, MT, Brasil. \\ ${ }^{2}$ Programa de Pós-Graduação em Agricultura Tropical, Universidade Federal de Mato Grosso, Cuiabá, MT, Brasil. \\ *E-mail: coelhomfstrela@gmail.com
}

(Orcid: 0000-0001-8878-3799; 0000-0003-1393-2504; 0000-0002-4642-2511)

Recebido em 02/06/2021; Aceito em 15/07/2021; Publicado em 28/07/2021.

\begin{abstract}
RESUMO: Os quintais urbanos têm sido suprimidos das moradias, alterando a qualidade de vida das pessoas. Em Cuiabá, além da destruição das casas antigas que possuíam extensos quintais, parte expressiva das novas moradias não apresentam espaços destinados ao cultivo de plantas. Objetivou-se neste estudo levantar as espécies vegetais mantidas nos quintais do bairro Nossa Senhora Aparecida em Cuiabá, Mato Grosso. Foram selecionados pela técnica de bola de neve 15 quintais, entrevistados os mantenedores e realizadas turnês guiadas com os mesmos para a coleta e identificação das espécies. A maioria dos entrevistados foi do sexo feminino e, as plantas foram obtidas com parentes e vizinhos. Mais de $60 \%$ das espécies são exóticas, mas, estão presentes plantas obtidas no Cerrado como bocaiúva (Acrocomia aculeata), mama-cadela (Brosimum gaudichaudii), assa-peixe (Vernonia polyanthes) e imbaúba (Cecropia pachystachya). Os quintais do bairro Nossa Senhora Aparecida têm grande diversidade vegetal, representada por 125 espécies pertencentes a 58 famílias botânicas. As famílias mais frequentes foram Lamiaceae, Asteraceae e Euphorbiaceae, com destaque para as espécies Annona squamosa L., Carica papaya L., Malpighia glabra L. e Mangifera indica L., todas frutíferas que contribuem para a segurança alimentar. O uso medicinal da maioria das espécies indica a importância do incentivo à manutenção da diversidade nos quintais.
\end{abstract}

Palavras-chave: diversidade; plantas medicinais; uso popular; segurança alimentar.

\section{Diversity and use of plants in homegardens in Nossa Senhora Aparecida district in Cuiabá, Mato Grosso, State, Brazil}

\begin{abstract}
Homegardens have been removed from housing, altering people's quality of life. In Cuiabá, in addition to the destruction of the old houses that had extensive homegardens, a significant part of the new houses do not have spaces for plants. The aim of this study was to survey the plant species kept in the homegardens of the Nossa Senhora Aparecida neighborhood in Cuiabá, Mato Grosso. 15 homegardens were selected by the snowball technique, the maintainers were interviewed and guided tours were held with them to collect and identify the species. Most of the interviewees were female and the plants were obtained from relatives and neighbors. More than $60 \%$ of the species are exotic, but there are plants obtained in the Cerrado such as bocaiúva (Acrocomia aculeata), mama-cadela (Brosimum gaudichaudii), assa-peixe (Vernonia polyanthes) and imbaúba (Cecropia pachystacbya). The homegardens of the Nossa Senhora Aparecida neighborhood have great plant diversity, represented by 125 species belonging to 58 botanical families. The most frequent families were Lamiaceae, Asteraceae and Euphorbiaceae, with emphasis on the species Annona squamosa L., Carica papaya L., Malpighia glabra L. and Mangifera indica L., all of which contribute to food security. The medicinal use of most species indicates the importance of encouraging the maintenance of diversity in homegardens.

Keywords: diversity; medicinal plants; popular use; food security.
\end{abstract}

\section{INTRODUÇÃO}

O crescimento urbano e desenvolvimento das cidades brasileiras tem resultado na eliminação dos espaços que mantêm grande diversidade de espécies vegetais como os quintais das residências. Em grandes cidades como Cuiabá/MT, além da destruição das casas antigas que possuíam extensos quintais, parte expressiva das novas moradias construídas não apresentam estes espaços.

Tourinho; Silva (2016) afirmaram que o conceito e funções dos quintais urbanos têm sido alterados ou, simplesmente, vêm sendo suprimidos das moradias e alertam para as consequências desse processo para a qualidade de vida urbana.
Os quintais urbanos são formas de uso da terra que se caracterizam por apresentar múltiplos estratos formados por espécies com diferentes hábitos assemelhando-se à estrutura de florestas tropicais, localizados ao redor das casas onde são feitos plantios de árvores, cultivo de grãos, hortaliças, plantas medicinais e ornamentais e, ainda, criação de animais, no mesmo espaço de terra (KUMAR; NAIR, 2006).

A importância dos quintais reflete na eficiência do uso da terra, conservação de espécies e sustentabilidade (KUMAR; NAIR, 2006; FLORENTINO et al., 2007), introdução e domesticação de espécies, segurança alimentar e aumento da renda familiar (GAO et al., 2012). Os quintais são 
considerados importantes na conservação de plantas medicinais por comunidades rurais e urbanas (BAJPAI et al., 2013).

Segundo Rondon Neto et al. (2004) a sustentabilidade está associada à diversidade de espécies, que permite a produção de alimento durante o ano todo, além de contemplar a distribuição mais equitativa de trabalho, comparado aos monocultivos agrícolas, além do que, possibilita o máximo aproveitamento de luz, nutrientes, espaço e água, atribuídos à diversidade de plantas. Um bom exemplo disto é o estudo conduzido por Vieira et al. (2013) em que os quintais de Bonito/PA são estabelecidos em pequenas áreas nas proximidades das moradias; a produção é destinada sobretudo à segurança alimentar e apenas o excedente é comercializado.

No Estado de Mato Grosso alguns estudos etnobotânicos foram realizados em quintais evidenciando a importância e os diferentes usos (AMARAL; GUARIM NETO, 2008, CARNIELLO et al., 2010, DE DAVID; PASA, 2015, MOREIRA; GUARIM NETO, 2015, PEREIRA; FIGUEIREDO NETO, 2015, AMARAL et al., 2016, SPILLER et al., 2016, SILVA et al., 2019). Infelizmente os quintais estão desaparecendo do cenário urbano, à medida que a especulação imobiliária avança com construção de edifícios com um mínimo de área verde para infiltração das águas de chuva, com pouca amenização do calor típico da região, além de reduzido espaço de convivência para a família e lazer das crianças.

Assim, no presente estudo objetivou-se realizar um levantamento das espécies vegetais presentes em quintais do Bairro Nossa Senhora Aparecida em Cuiabá, Mato Grosso.

\section{MATERIAL E MÉTODOS}

O município de Cuiabá situa-se na região Centro Sul do estado de Mato Grosso, nas coordenadas 15035'46" S e $56^{\circ} 05^{\prime} 48^{\prime \prime} \mathrm{W}$ com população de 612.597 habitantes, segundo o censo de 2019 (IBGE, 2019).

O clima é tropical chuvoso e, segundo Koppen é classificado como Am, com duas estações bem definidas, verão chuvoso e inverno seco e, apresenta elevado índice pluviométrico no verão podendo atingir médias superiores a $1.300 \mathrm{~mm}$ com intensidade máxima em janeiro, fevereiro e março. A temperatura máxima pode chegar aos $40{ }^{\circ} \mathrm{C}$ nos meses mais quentes. A média da mínima em julho, o mês mais frio, é de $16,6^{\circ} \mathrm{C}$. A altitude é de 146 a $250 \mathrm{~m}$ acima do nível do mar (ALVARES et al., 2014; IBGE, 2019).

O presente estudo foi realizado em quinze quintais do bairro Nossa Senhora Aparecida em Cuiabá/MT. Na seleção dos entrevistados foi aplicada a técnica de amostragem bola de neve, ou seja, iniciou-se a entrevista com a pessoa responsável por um quintal, escolhida aleatoriamente e, no final da entrevista solicitou-se que o entrevistado indicasse outra pessoa e assim, sucessivamente (ALBUQUERQUE et al., 2014). Foram usadas as técnicas de observação direta e participante e, os principais envolvidos na manutenção dos quintais foram indagados sobre o manejo das plantas e as partes empregadas para cada uso, além do perfil socioeconômico (idade, escolaridade, origem, tempo de residência, etc.) (ALBUQUERQUE et al., 2014).

A verificação in loco das espécies citadas pelos entrevistados foi realizada utilizando-se o método turnê guiada, que consiste em ir até a área com o informante visando fundamentar e validar os nomes das plantas citadas nas entrevistas (ALBUQUERQUE et al., 2014).

Em cada quintal visitado foram feitos levantamentos das espécies vegetais com a coleta de amostras para exsicatas e identificação no Herbário da Universidade Federal de Mato Grosso. As informações foram compiladas sob a forma de um banco de dados, utilizando-se matrizes de textos, conforme metodologia proposta por AMOROZO; VIERTLER (2010).

\section{RESULTADOS}

O bairro Nossa Senhora Aparecida localiza-se na região sul da cidade de Cuiabá/MT, e é considerado de renda média. Em entrevista com um dos moradores antigos verificou-se que a construção do bairro teve início na década de 1970 com os lotes demarcados pela Imobiliária Mato Grosso. Em 1974 esta imobiliária vendeu o direito para a Imobiliária Petrópolis, ficando esta responsável pela venda dos terrenos. A partir do ano de 1975 a Caixa Econômica Federal liberou um financiamento, a quem detinha a escritura dos terrenos, para construção das casas. No início a construção serviu para abrigar os trabalhadores da Empresa Cuiabá Diesel que tinham certo grau de parentesco e eram paulistas e goianos, e este fato influenciou na arquitetura padrão das casas inicialmente construídas.

A partir da década de 1980 desenvolveu-se no bairro outra arquitetura, tanto das novas construções como da reforma das existentes, devido à vinda de pessoas de outras origens e de maior poder aquisitivo, que compraram terrenos neste local. No bairro não tem posto policial nem posto de saúde, apenas uma Escola Estadual, duas áreas de lazer, sendo uma quadra na escola e outra em uma área verde destinada à praça do bairro. A Associação de Moradores possui uma diretoria com mandato de dois anos.

Os quintais apresentaram forma retangular e área média de $360 \mathrm{~m}^{2}$. A idade média de formação dos quintais é de 15 anos (Figura 1A), o número médio de pessoas por família é igual a três (Figura 1B) e na maioria dos quintais a predominância de entrevistados é do sexo feminino (60\%).

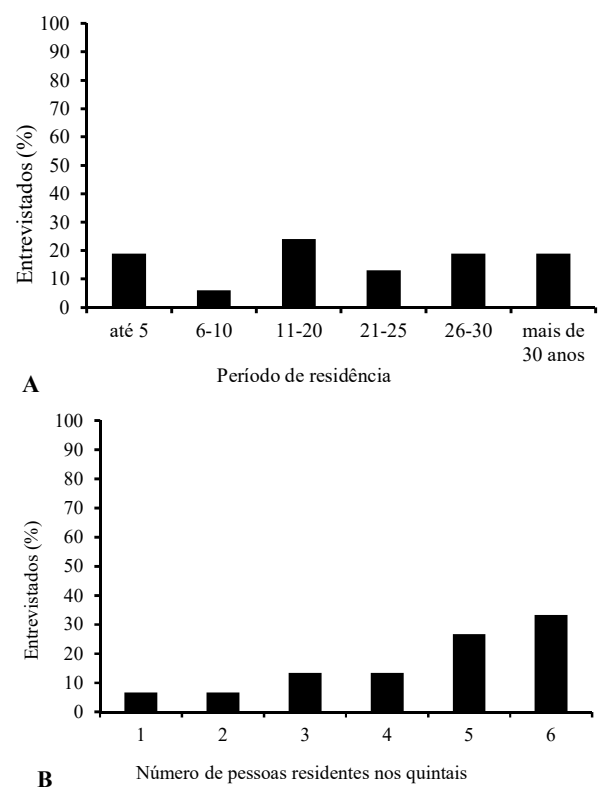

Figura 1. Período de residência dos entrevistados responsáveis pela manutenção dos quintais (A) e número de pessoas residentes (B) no bairro Nossa Senhora Aparecida em Cuiabá, Mato Grosso. 
Figure 1. Period of residence of respondents responsible for maintaining the homegardens (A) and number of people living (B) at Nossa Senhora Aparecida district, Cuiabá, Mato Grosso.
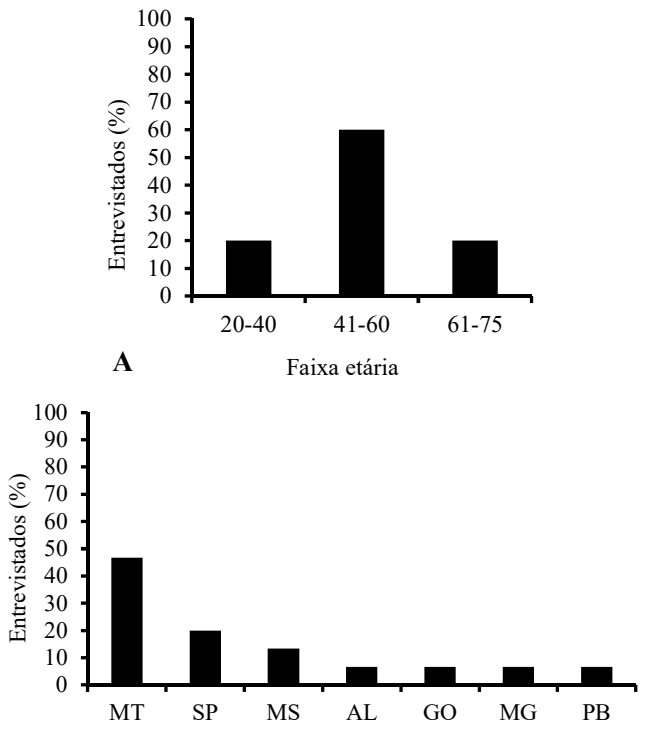

Estados brasileiros

Figura 2. Faixa etária (A) e origem (B) dos entrevistados responsáveis pela manutenção dos quintais no bairro Nossa Senhora Aparecida em Cuiabá, Mato Grosso.

Figure 2. Age (A) and origin (B) of respondents responsible for maintaining homegardens at Nossa Senhora Aparecida district, Cuiabá, Mato Grosso.
A faixa etária dos quinze informantes entrevistados nos quintais do bairro Nossa Senhora Aparecida predomina entre 41 e 60 anos, representando $60 \%$, como verificado na Figura 2A. Nos quintais estudados o maior número dos entrevistados é Mato-grossense, seguido por pessoas vindas de São Paulo e Mato Grosso do Sul e, outros quatro estados (Figura 2B).

Verificou-se que $40 \%$ dos entrevistados afirmaram que a responsabilidade da escolha das espécies, do plantio e manutenção dos quintais é tanto do homem como da mulher, no caso, esposo e esposa (Figura 3). A diversidade vegetal nos quinze quintais do bairro Nossa Senhora Aparecida está representada por 125 espécies e 58 famílias botânicas (Tabela $1)$.

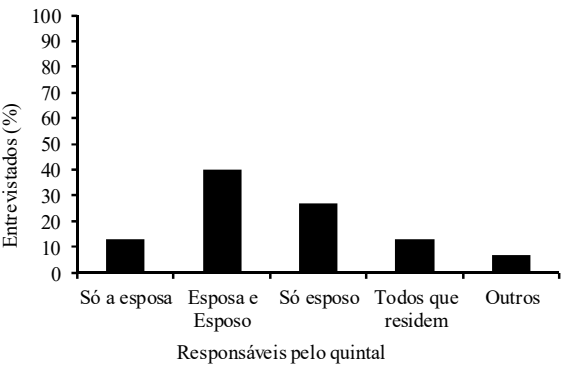

Figura 3. Responsáveis pela manutenção dos quintais no bairro Nossa Senhora Aparecida em Cuiabá, Mato Grosso.

Figure 3. Those responsible for maintaining the homegardes at Nossa Senhora Aparecida district, Cuiabá, Mato Grosso.

Tabela 1. Espécies, origem $(\mathrm{N}=$ nativa, $\mathrm{E}=$ exótica), uso, hábito de crescimento e frequência (\%) em quintais no Bairro Nossa Senhora Aparecida em Cuiabá, Mato Grosso.

Table 1. Species, origin $(\mathrm{N}=$ native, $\mathrm{E}=$ exotic), use, growth habit and frequency $(\%)$ in homegardens at Nossa Senhora Aparecida district, Cuiabá, Mato Grosso

\begin{tabular}{|c|c|c|c|c|c|}
\hline Família/Nome científico & Nome vernacular & Origem & Uso* $^{*}$ & Hábito de crescimento & Freq. \\
\hline $\begin{array}{l}\text { Acantaceae } \\
\text { Justicia pectoralis var. stenophylla Leon. }\end{array}$ & Anador & $\mathrm{N}$ & med & herbáceo & 13,3 \\
\hline $\begin{array}{l}\text { Alismataceae } \\
\text { Echinodorus grandiflorus Mitch. }\end{array}$ & Chapéu de couro & $\mathrm{N}$ & med & herbáceo & 6,7 \\
\hline $\begin{array}{l}\text { Amaranthaceae } \\
\text { Amaranthus deflexus } \mathrm{L} . \\
\text { Celosia argentea } \mathrm{L} . \\
\text { Alternanthera brasiliana (L.) O. Kuntze }\end{array}$ & $\begin{array}{c}\text { Caruru } \\
\text { Crista-de-galo-plumosa } \\
\text { Penicilina }\end{array}$ & $\begin{array}{l}N \\
E \\
N\end{array}$ & $\begin{array}{l}\text { ali } \\
\text { orn } \\
\text { med }\end{array}$ & $\begin{array}{l}\text { herbáceo } \\
\text { herbáceo } \\
\text { herbáceo }\end{array}$ & $\begin{array}{l}6,7 \\
6,7 \\
13,3\end{array}$ \\
\hline $\begin{array}{l}\text { Amaryllidaceae } \\
\text { Zephyranthes rosea Lindl. }\end{array}$ & Lírio do zéfiro & $\mathrm{E}$ & orn & herbáceo & 6,7 \\
\hline $\begin{array}{l}\text { Anacardiaceae } \\
\text { Spondias dulcis Fort. } \\
\text { Anacardium occidentale } \mathrm{L} . \\
\text { Mangifera indica } \mathrm{L} . \\
\text { Spondias mombin } \mathrm{L} .\end{array}$ & $\begin{array}{l}\text { Cajarana } \\
\text { Cajueiro } \\
\text { Mangueira } \\
\text { Cajá }\end{array}$ & $\begin{array}{l}N \\
E \\
E \\
N\end{array}$ & $\begin{array}{l}\text { frut } \\
\text { frut } \\
\text { frut } \\
\text { frut }\end{array}$ & $\begin{array}{l}\text { arbóreo } \\
\text { arbóreo } \\
\text { arbóreo } \\
\text { arbóreo }\end{array}$ & $\begin{array}{l}13,3 \\
33,3 \\
66,7 \\
6,7\end{array}$ \\
\hline $\begin{array}{l}\text { Annonaceae } \\
\text { Annona squamosa } \mathrm{L} \text {. } \\
\text { Annona muricata } \mathrm{L} \text {. }\end{array}$ & $\begin{array}{l}\text { Ata } \\
\text { Graviola }\end{array}$ & $\begin{array}{l}\mathrm{N} \\
\mathrm{E}\end{array}$ & $\begin{array}{l}\text { frut } \\
\text { frut }\end{array}$ & $\begin{array}{l}\text { arbóreo } \\
\text { arbóreo }\end{array}$ & $\begin{array}{l}66,7 \\
6,7\end{array}$ \\
\hline $\begin{array}{l}\text { Apiaceae } \\
\text { Coriandrum sativum L. } \\
\text { Petroselinum sativum Hoffm. } \\
\text { Foeniculum vulgare Mill. } \\
\end{array}$ & $\begin{array}{l}\text { Coentro } \\
\text { Salsa } \\
\text { Erva doce }\end{array}$ & $\begin{array}{l}E \\
E \\
E\end{array}$ & $\begin{array}{l}\text { cond } \\
\text { cond } \\
\text { med }\end{array}$ & $\begin{array}{l}\text { herbáceo } \\
\text { herbáceo } \\
\text { herbáceo }\end{array}$ & $\begin{array}{l}6,7 \\
6,7 \\
6,7 \\
\end{array}$ \\
\hline $\begin{array}{l}\text { Apocinaceae } \\
\text { Catharanthus roseus (L) G. Don } \\
\text { Carissa macrocarpa (Eckl.) A. DC. }\end{array}$ & $\begin{array}{l}\text { Boa noite } \\
\text { Laranjinha }\end{array}$ & $\begin{array}{l}E \\
E\end{array}$ & $\begin{array}{l}\text { orn } \\
\text { orn }\end{array}$ & $\begin{array}{l}\text { herbáceo } \\
\text { arbustivo }\end{array}$ & $\begin{array}{l}6,7 \\
6,7\end{array}$ \\
\hline $\begin{array}{l}\text { Araceae } \\
\text { Spathiphyllum cannifolium (Dryand.) Schott } \\
\text { Dieffenbachia amoena Bull. } \\
\text { Caladium x hortulanum Birdsey } \\
\text { Anthurium andraeanum Linden } \\
\text { Spathiphyllum wanllisi Regel } \\
\text { Anthurium spectabile Schott. }\end{array}$ & $\begin{array}{c}\text { Bandeira branca } \\
\text { Comigo-ninguém-pode } \\
\text { Coração-de-Jesus } \\
\text { Antúrio } \\
\text { Lírio da paz } \\
\text { Taioba }\end{array}$ & $\begin{array}{l}N \\
N \\
E \\
E \\
E \\
N\end{array}$ & $\begin{array}{l}\text { orn } \\
\text { prot } \\
\text { orn } \\
\text { orn } \\
\text { orn } \\
\text { ali }\end{array}$ & $\begin{array}{l}\text { herbáceo } \\
\text { herbáceo } \\
\text { herbáceo } \\
\text { herbáceo } \\
\text { herbáceo } \\
\text { herbáceo }\end{array}$ & $\begin{array}{l}6,7 \\
13,3 \\
6,7 \\
20\end{array}$ \\
\hline
\end{tabular}


Tabela 1. Espécies, origem ( $\mathrm{N}=$ nativa, $\mathrm{E}=$ exótica), uso, hábito de crescimento e frequência (\%) em quintais ... (CONTINUAÇÃO)

Table 1. Species, origin $(\mathrm{N}=$ native, $\mathrm{E}=$ exotic), use, growth habit and frequency $(\%)$ in homegardens ... (CONTINUATION)

\begin{tabular}{|c|c|c|c|c|c|}
\hline Família/Nome científico & Nome vernacular & Origem & Uso* & Hábito de crescimento & Freq. \\
\hline \multicolumn{6}{|l|}{ Arecaceae } \\
\hline Acrocomia aculeata (Jacq.) Lodd. & Bocaiúva & $\mathrm{N}$ & ali & arbustivo & 20 \\
\hline Cocos nucifera $\mathrm{L}$. & Coqueiro & $\mathrm{E}$ & ali & arbustivo & 20 \\
\hline Syagrus oleracea (Mart.) Becc. & Gueroba & $\mathrm{N}$ & ali & arbustivo & 6,7 \\
\hline Dypsis lutescens (H. Wendl.) Beentje \& J. Dransf. & Palmeira areca & $\mathrm{E}$ & orn & arbustivo & 26,7 \\
\hline \multicolumn{6}{|l|}{ Aristoloquiaceae } \\
\hline Aristolochia triangularis $\mathrm{Cham}$. & Cipó mil homens & $\mathrm{N}$ & med & herbáceo & 6,7 \\
\hline \multicolumn{6}{|l|}{ Asteraceae } \\
\hline Artemisia vulgaris $\mathrm{L}$. & Artemísia & $\mathrm{E}$ & med & herbáceo & 6,7 \\
\hline Vernonia polyanthes Less. & Assapeixe & $\mathrm{N}$ & med & arbustivo & 26,7 \\
\hline Vernonia condensata Baker & Boldo chinês & $\mathrm{N}$ & med & arbustivo & 13,3 \\
\hline Tanacetum parthenium (L.) Sch. Bip. & Camomila pequena & $\mathrm{E}$ & med & herbáceo & 20 \\
\hline Acanthospermum australe (Loefl.) Kuntze & Carrapicho rasteiro & $\mathrm{N}$ & med & herbáceo & 6,7 \\
\hline Artemisia absintbium $\mathrm{L}$ & Losna & $\mathrm{E}$ & med & herbáceo & 20 \\
\hline Bidens pilosa $\mathrm{L}$. & Picão & $\mathrm{N}$ & med & herbáceo & 6,7 \\
\hline Baccharis dracunculifolia DC. & Vassourinha & $\mathrm{N}$ & med & herbáceo & 6,7 \\
\hline \multicolumn{6}{|l|}{ Bignoniaceae } \\
\hline Tabebuia avellanedae Lor. Ex Griseb. & Ipê rosa & $\mathrm{N}$ & orn & arbóreo & 6,7 \\
\hline \multicolumn{6}{|l|}{ Bixaceae } \\
\hline Bixa orellana $\mathrm{L}$. & Urucum & $\mathrm{N}$ & med & arbustivo & 6,7 \\
\hline \multicolumn{6}{|l|}{ Borraginaceae } \\
\hline Symphytum officinale L. & Confrei & $\mathrm{N}$ & med & herbáceo & 6,7 \\
\hline \multicolumn{6}{|l|}{ Bromeliaceae } \\
\hline Ananas sativus Schult. & Abacaxi & $\mathrm{N}$ & frut & herbáceo & 6,7 \\
\hline \multicolumn{6}{|l|}{ Caesalpiniaceae } \\
\hline Caesalpinia peltophoroides Benth. & Sibipiruna & $\mathrm{N}$ & orn & arbóreo & 6,7 \\
\hline \multicolumn{6}{|l|}{ Caprifoliaceae } \\
\hline Sambucus australis Cham. \& Schltdl. & Sabugueiro & $\mathrm{N}$ & med & arbustivo & 6,7 \\
\hline \multicolumn{6}{|l|}{ Caricaceae } \\
\hline Carica papaya Lin. & Mamoeiro & $\mathrm{E}$ & frut & arbustivo & 66,7 \\
\hline \multicolumn{6}{|l|}{ Cecropiaceae } \\
\hline Cecropia pachystachya Trec. & Imbaúba & $\mathrm{N}$ & med & arbóreo & 13,3 \\
\hline \multicolumn{6}{|l|}{ Chenopodiaceae } \\
\hline Chenopodium ambrosioides $\mathrm{L}$. & Erva de Santa Maria & $\mathrm{E}$ & med & herbáceo & 26,7 \\
\hline \multicolumn{6}{|l|}{ Convolvulaceae } \\
\hline Ipomoea batatas L. Lam. & Batata doce & $\mathrm{N}$ & ali & herbáceo & 6,7 \\
\hline \multicolumn{6}{|l|}{ Costaceae } \\
\hline Costus spicatus (Jacq.) Sw. & Cana de macaco & $\mathrm{E}$ & med & arbustivo & 26,7 \\
\hline Crassulaceae & & & & & \\
\hline Crassula argentea $\mathrm{L}$. & Balsamo & $\mathrm{N}$ & med & herbáceo & 6,7 \\
\hline Kalanchoe brasiliensis St. Hil. & Folha da fortuna & $\mathrm{N}$ & med & herbáceo & 33,3 \\
\hline Brassica oleracea $L$. & Couve & $\mathrm{E}$ & ali & herbáceo & 13,3 \\
\hline Cucurbitaceae & & & & & \\
\hline Curcubita pepo L. & Abóbora & $\mathrm{E}$ & ali & herbáceo & 6,7 \\
\hline Cupressaceae & & & & & \\
\hline Chamaecyparis obtusa Endl. Var. cripssii Rehder & Pinheiro dourado & $\mathrm{E}$ & orn & arbustivo & 6,7 \\
\hline Cycadaceae & & & & & \\
\hline Cycas revoluta Thunb. & Cica & $\mathrm{E}$ & orn & arbustivo & 6,7 \\
\hline Davalliaceae & & & & & \\
\hline Nephrolepis exaltata (L.) schott & Samambaia espada & $\mathrm{E}$ & orn & herbáceo & 40 \\
\hline Dioscoreaceae & & & & & \\
\hline Dioscorea petrea A. Penna & Cará & $\mathrm{N}$ & ali & herbáceo & 6,7 \\
\hline Euphorbiaceae & & & & & \\
\hline Euphorbia pucherrima Willd. Ex Klotzsch & Bico de papagaio & $\mathrm{E}$ & orn & arbustivo & 6,7 \\
\hline Jatropha gossypiifolia $L$ & Pião roxo & $\mathrm{E}$ & prot & arbustivo & 6,7 \\
\hline Pedilanthus tithymaloides (L.) Poit. & Sapatinho dos jardins & $\mathrm{E}$ & orn & arbustivo & 6,7 \\
\hline Ricinus communis $L$. & Mamona & $\mathrm{E}$ & med & arbustivo & 13,3 \\
\hline Manihot esculenta Crantz. & Mandioca & $\mathrm{N}$ & ali & arbustivo & 13,3 \\
\hline Phyllanthus niruri L. & Quebra pedra & $\mathrm{N}$ & med & herbáceo & 20 \\
\hline Euphorbia milii Dês Moul. & Coroa de Cristo & $\mathrm{E}$ & orn & herbáceo & 6,7 \\
\hline Fabaceae & & & & & \\
\hline Vigna unguiculata L. Walp. & Feijão miúdo & $\mathrm{E}$ & ali & herbáceo & 13,3 \\
\hline Gesneriaceae & & & & & \\
\hline Saintpaulia ionantha Wendl. & Violeta & $\mathrm{E}$ & orn & herbáceo & 6,7 \\
\hline
\end{tabular}


Silva et al.

Tabela 1. Espécies, origem ( $\mathrm{N}=$ nativa, $\mathrm{E}=$ exótica), uso, hábito de crescimento e frequência (\%) em quintais ... (CONTINUAÇ̃̃)

Table 1. Species, origin $(\mathrm{N}=$ native, $\mathrm{E}=$ exotic), use, growth habit and frequency $(\%)$ in homegardens ... (CONTINUATION)

\begin{tabular}{|c|c|c|c|c|c|}
\hline Família/Nome científico & Nome vernacular & Origem & Uso* & Hábito de crescimento & Freq. \\
\hline \multicolumn{6}{|l|}{ Lamiaceae } \\
\hline Rosmarinus officinalis $\mathrm{L}$. & Alecrim & $\mathrm{E}$ & med & herbáceo & 6,7 \\
\hline Hyptis suaveolens (L.) Poit. & Alfazema & $\mathrm{N}$ & med & herbáceo & 6,7 \\
\hline Plectranthus barbatus Andrews & Boldo & $\mathrm{E}$ & med & arbustivo & 33,3 \\
\hline Leucas martinicensis (Jacq.) R.Br. & Cordão de São Francisco & $\mathrm{N}$ & med & herbáceo & 13,3 \\
\hline Mentha $x$ piperita L. & Hortelã & $\mathrm{E}$ & med & herbáceo & 6,7 \\
\hline Plectranthus amboinicus (Lour.) Spreng. & Hortelã pimenta & $\mathrm{N}$ & med & herbáceo & 20 \\
\hline Ocimum basilicum $\mathrm{L}$. & Manjericão & $\mathrm{E}$ & med & herbáceo & 26,7 \\
\hline Mentha pulegium L. & Poejo & $\mathrm{E}$ & med & herbáceo & 6,7 \\
\hline Ocimum gratissimum $\mathrm{L}$. & Alfavaca & $\mathrm{E}$ & med & herbáceo & 40 \\
\hline \multicolumn{6}{|l|}{ Lauraceae } \\
\hline Cinnamomum zeylanicum Bryn. & Canela & $\mathrm{E}$ & med & arbóreo & 6,7 \\
\hline \multicolumn{6}{|l|}{ Liliaceae } \\
\hline Dracaena sanderiana Hort. & Dracena fita & $\mathrm{E}$ & orn & arbustivo & 6,7 \\
\hline Hemerocallis flava $\mathrm{L}$. & Lírio de São José & $\mathrm{N}$ & orn & herbáceo & 6,7 \\
\hline Aloe arborecens Mill. & Babosa & $\mathrm{N}$ & med & herbáceo & 53,3 \\
\hline Allium fistulosum $\mathrm{L}$. & Cebolinha verde & $\mathrm{E}$ & cond & herbáceo & 53,3 \\
\hline Sansevieria trifasciata var. laurentii (De Wild.) N.E.Br & Espada-de-São Jorge & $\mathrm{E}$ & prot & herbáceo & 13,3 \\
\hline Smilax brasiliensis Spreng. & Japecanga & $\mathrm{N}$ & med & herbáceo & 6,7 \\
\hline \multicolumn{6}{|l|}{ Malpighiaceae } \\
\hline Malpighia glabra L. & Acerola & $\mathrm{E}$ & frut & arbóreo & 66,7 \\
\hline \multicolumn{6}{|l|}{ Malvaceae } \\
\hline Gossypium barbadense $\mathrm{L}$. & Algodão & $\mathrm{E}$ & med & arbustivo & 20 \\
\hline Hibiscus rosa-sinensis $\mathrm{L}$. & Hibisco tropical & $\mathrm{E}$ & orn & arbustivo & 6,7 \\
\hline Hibiscus mutabilis $\mathrm{L}$ & Rosa branca & $\mathrm{E}$ & orn & arbustivo & 26,7 \\
\hline \multicolumn{6}{|l|}{ Mimosaceae } \\
\hline Inga uruguensis Hook. \& arn. & Ingazeira & $\mathrm{N}$ & frut & arbóreo & 13,3 \\
\hline \multicolumn{6}{|l|}{ Mirtaceae } \\
\hline Psidium guajava $\mathrm{L}$. & Goiaba & $\mathrm{E}$ & frut & arbóreo & 53,3 \\
\hline Myrciaria trunciflora Berg. & Jabuticaba & $\mathrm{N}$ & frut & arbóreo & 33,3 \\
\hline Eugenia uniflora $\mathrm{L}$. & Pitanga & $\mathrm{N}$ & frut & arbóreo & 20 \\
\hline \multicolumn{6}{|l|}{ Moraceae } \\
\hline Artocarpus integrifolia Forst. & Jaqueira & $\mathrm{E}$ & frut & arbóreo & 6,7 \\
\hline Brosimum gaudichaudii Trécul & Maminha cadela & $\mathrm{N}$ & med & arbóreo & 6,7 \\
\hline \multicolumn{6}{|l|}{ Musaceae } \\
\hline Musa paradisiaca $\mathrm{L}$. & Banana & $\mathrm{E}$ & frut & arbustivo & 46,7 \\
\hline \multicolumn{6}{|l|}{ Nyctaginaceae } \\
\hline Mirabilis jalapa $\mathrm{L}$. & Maravilha & $\mathrm{N}$ & med & herbáceo & 6,7 \\
\hline \multicolumn{6}{|l|}{ Orchidaceae } \\
\hline X BLC"Luck strike" & Orquídea & $\mathrm{E}$ & orn & herbáceo & 20 \\
\hline Oncidium varicosum Lindl. Ex Paxton & Chuva de ouro & $\mathrm{N}$ & orn & arbóreo & 6,7 \\
\hline Oxalidaceae & & & & & \\
\hline Oxalis vulcanicola Donn. Sm. & Trevo & $\mathrm{N}$ & orn & herbáceo & 6,7 \\
\hline Plantaginaceae & & & & & \\
\hline Plantago major $\mathrm{L}$. & Tansagem & $\mathrm{E}$ & med & herbáceo & 13,3 \\
\hline Poaceae & & & & & \\
\hline Cymbopogon citratus (DC) Stapf & Capim cidreira & E & med & arbustivo & 26,7 \\
\hline Poligonaceae & & & & & \\
\hline Polygonum bydropiper L. & Erva de bicho & $\mathrm{N}$ & med & herbáceo & 6,7 \\
\hline Polipodiaceae & & & & & \\
\hline Adiantum capillus veneris & Avenca & $\mathrm{E}$ & orn & herbáceo & 6,7 \\
\hline Portulacaceae & & & & & \\
\hline Portulaca oleracea $L$. & Ora-pro-nobis & $\mathrm{N}$ & ali & arbustivo & 6,7 \\
\hline Punicaceae & & & & & \\
\hline Punica granatum $L$. & Romã & $\mathrm{E}$ & med & arbustivo & 13,3 \\
\hline Rosaceae & & & & & \\
\hline Rosa wichuraiana Crép. & Rosa trepadeira & $\mathrm{E}$ & orn & arbustivo & 33,3 \\
\hline Prunus domestica L. & Ameixa & $\mathrm{E}$ & frut & arbustiva & 6,7 \\
\hline Rubus fruticosus $L$. & Amora preta & $\mathrm{N}$ & frut & arbustivo & 6,7 \\
\hline Rubiaceae & & & & & \\
\hline Ixora coccinea L. "Compacta" & Ixora compacta & $\mathrm{E}$ & orn & arbustivo & 20 \\
\hline Mussaenda erytbrophylla Schumanch. \& Thonn. & Mussanda rosa & $\mathrm{E}$ & orn & arbustivo & 13,3 \\
\hline Rutaceae & & & & & \\
\hline Ruta graveolens $L$. & Arruda & $\mathrm{E}$ & med & herbáceo & 20 \\
\hline Pilocarpus microphyllus Stapf ex Wardleworth & Jaborandi & $\mathrm{N}$ & med & arbustivo & 6,7 \\
\hline Citrus aurantium $L$. & Laranja azeda & $\mathrm{E}$ & frut & arbóreo & 6,7 \\
\hline Citrus sinensis $L$. & Laranja lima & $\mathrm{E}$ & frut & arbóreo & 20 \\
\hline Citrus limon (L.) Burm. & Limão galego & $\mathrm{E}$ & frut & arbóreo & 33,3 \\
\hline Citrus reticulata & Ponkan & $\mathrm{E}$ & frut & arbóreo & 6,7 \\
\hline
\end{tabular}


Tabela 1. Espécies, origem ( $\mathrm{N}=$ nativa, $\mathrm{E}=$ exótica), uso, hábito de crescimento e frequência (\%) em quintais ... (CONTINUAÇÃO)

Table 1. Species, origin ( $\mathrm{N}=$ native, $\mathrm{E}=$ exotic), use, growth habit and frequency (\%) in homegardens ... (CONTINUATION)

\begin{tabular}{|c|c|c|c|c|c|}
\hline Família/Nome científico & Nome vernacular & Origem & Uso* & Hábito de crescimento & Freq. \\
\hline \multicolumn{6}{|l|}{ Solanaceae } \\
\hline Cestrum nocturnum $\mathrm{L}$. & Dama da noite & $\mathrm{E}$ & orn & arbustivo & 13,3 \\
\hline Nicotiana tabacum $\mathrm{L}$. & Fumo & $\mathrm{N}$ & ins & arbustivo & 6,7 \\
\hline Solanum paniculatum L. & Jurubeba & $\mathrm{N}$ & med & arbustivo & 13,3 \\
\hline Capsicum frutescens $\mathrm{L}$. & Pimenta & $\mathrm{N}$ & cond & herbáceo & 33,3 \\
\hline Capsicum annuum $\mathrm{L}$. & Pimentão & $\mathrm{E}$ & ali & herbáceo & 6,7 \\
\hline Lycopersicum esculentum $\mathrm{L}$. & Tomate & $\mathrm{E}$ & ali & herbáceo & 6,7 \\
\hline \multicolumn{6}{|l|}{ Verbenaceae } \\
\hline Lippia alba (Mill.) N.E. Br. & Erva cidreira & $\mathrm{N}$ & med & arbustivo & 46,7 \\
\hline Duranta repens $\mathrm{L}$. & Pingo de ouro & $\mathrm{E}$ & orn & arbóreo & 20 \\
\hline \multicolumn{6}{|l|}{ Vitaceae } \\
\hline Vitis vinifera $\mathrm{L}$. & Uva & $\mathrm{E}$ & frut & herbáceo & 13,3 \\
\hline Cissus verticillata (L.) Nicholson \& C.E.Jarvis & Insulina & $\mathrm{E}$ & med & herbáceo & 6,7 \\
\hline \multicolumn{6}{|l|}{ Zingiberaceae } \\
\hline Curcuma longa $\mathrm{L}$. & Açafrão & $\mathrm{E}$ & med & herbáceo & 13,3 \\
\hline Alpinia zerumbet (Pers.) B.L. Burtt. \& R.M. Sm. & Colônia & $\mathrm{E}$ & med & arbustivo & 13,3 \\
\hline Zingiber officinale Roscoe. & Gengibre & $\mathrm{E}$ & med & herbáceo & 6,7 \\
\hline
\end{tabular}

*Ali $=$ alimentício, Cond $=$ condimentar, Frut $=$ frutífero, Ins $=$ inseticida, Med $=$ medicinal, Orn $=$ ornamental, Prot $=$ proteção

$*$ Ali $=$ food, Cond $=$ spice, Fruit $=$ fruit, Ins $=$ insecticide, Med $=$ medicinal, Orn $=$ ornamental, Prot $=$ protection

As famílias mais frequentes nos quintais foram Lamiaceae, Asteraceae e Euphorbiaceae (Figura 4).

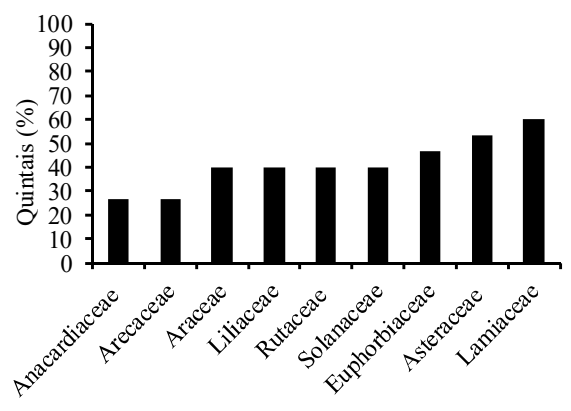

Famílias mais frequentes

Figura 4. Famílias botânicas mais frequentes nos quintais do bairro Nossa Senhora Aparecida em Cuiabá, Mato Grosso.

Figure 4. Most frequent botanical families in the homegardens at Nossa Senhora Aparecida district, Cuiabá, Mato Grosso.
Os entrevistados classificaram as espécies dos quintais em inseticida, proteção, condimentar, alimentício, frutífera, ornamental e medicinal, com maior percentual desta última categoria (Figura 5). O uso medicinal está presente em todos os quintais estudados (Tabela 2).

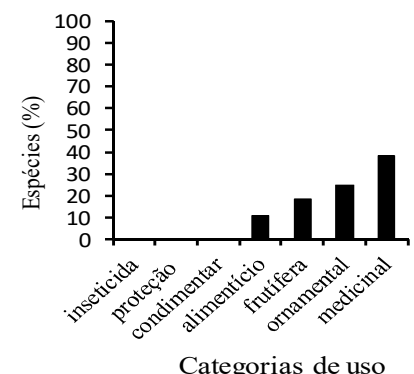

Figura 5. Categorias de uso das espécies nos quintais no bairro Nossa Senhora Aparecida em Cuiabá, Mato Grosso.

Figure 5. Categories of species use in homegardens at Nossa Senhora Aparecida district, Cuiabá, Mato Grosso.

Tabela 2. Espécies de uso medicinal no bairro Nossa Senhora Aparecida, Cuiabá, Mato Grosso.

Table 2. Species of medicinal use at Nossa Senhora Aparecida district, Cuiabá, Mato Grosso.

\begin{tabular}{|c|c|c|}
\hline Família/Nome científico & Nome vernacular & Usos \\
\hline $\begin{array}{l}\text { Acantaceae } \\
\text { Justicia pectoralis var. stenophylla Leon. }\end{array}$ & Anador & Chá para dor de cabeça, gripe, mal estar \\
\hline $\begin{array}{l}\text { Alismataceae } \\
\text { Echinodorus grandiflorus Mitch. }\end{array}$ & Chapéu-de-couro & Chá para o fígado e rins \\
\hline $\begin{array}{l}\text { Amaranthaceae } \\
\text { Alternanthera brasiliana (L.) O. Kuntze }\end{array}$ & Penicilina & Chá das folhas para dor, febre e infecções \\
\hline $\begin{array}{l}\text { Apiaceae } \\
\text { Foeniculum vulgare Mill. }\end{array}$ & Erva doce & Chá das sementes para digestão, dor de estomago, gases \\
\hline $\begin{array}{l}\text { Aristoloquiaceae } \\
\text { Aristolochia triangularis Cham. }\end{array}$ & Cipó mil homens & Chá para o estômago, fígado e raiz contra picada de cobra \\
\hline $\begin{array}{l}\text { Asteraceae } \\
\text { Artemisia vulgaris } \mathrm{L} . \\
\text { Vernonia polyanthes Less. } \\
\text { Vernonia condensata Baker } \\
\text { Tanacetum parthenium (L.) Sch. Bip. } \\
\text { Acanthospermum australe Kuntze } \\
\text { Artemisia absinthium } \mathrm{L} \\
\text { Bidens pilosa } \mathrm{L} . \\
\text { Baccharis dracunculifolia } \mathrm{DC} . \\
\end{array}$ & $\begin{array}{l}\text { Artemísia } \\
\text { Assa-peixe } \\
\text { Boldo chinês } \\
\text { Camomila pequena } \\
\text { Carrapicho rasteiro } \\
\text { Losna } \\
\text { Picão } \\
\text { Vassourinha } \\
\end{array}$ & $\begin{array}{l}\text { Chá para gases, azia e diarréia } \\
\text { Chá para bronquite e asma } \\
\text { Chá para azia, dor de cabeça e má digestão } \\
\text { Chá para dor de cabeça } \\
\text { Chá para gripe } \\
\text { Chá para cólicas intestinais e verminoses } \\
\text { Chá para icterícia } \\
\text { Chá para fraqueza e reumatismo }\end{array}$ \\
\hline $\begin{array}{l}\text { Bixaceae } \\
\text { Bixa orellana } \mathrm{L} .\end{array}$ & Urucum & Chá das folhas como cicatrizante \\
\hline $\begin{array}{l}\text { Borraginaceae } \\
\text { Symphytum officinale } \mathrm{L} \text {. }\end{array}$ & Confrei & Amassar as folhas para cicatrizar ferimentos \\
\hline
\end{tabular}

Nativa, Sinop, v. 9, n. 3, p. 327-336, mai./jun. 2021. 
Tabela 2. Espécies de uso medicinal no bairro Nossa Senhora Aparecida, Cuiabá, Mato Grosso.

Table 2. Species of medicinal use at Nossa Senhora Aparecida district, Cuiabá, Mato Grosso.

\begin{tabular}{|c|c|c|}
\hline Família/Nome científico & Nome vernacular & Usos \\
\hline \multicolumn{3}{|l|}{ Caprifoliaceae } \\
\hline Sambucus australis Cham. \& Schltdl. & Sabugueiro & Chá das flores para sarampo \\
\hline \multicolumn{3}{|l|}{ Cecropiaceae } \\
\hline Cecropia pachystachya Trec. & Imbaúba & Chá das flores para pressão alta \\
\hline \multicolumn{3}{|l|}{ Chenopodiaceae } \\
\hline Chenopodium ambrosioides L. & Erva de Santa Maria & Sumo das folhas para vermes \\
\hline \multicolumn{3}{|l|}{ Costaceae } \\
\hline Costus spicatus (Jacq.) Sw. & Cana de macaco & Chá para inflamação nos rins \\
\hline \multicolumn{3}{|l|}{ Crassulaceae } \\
\hline Crassula argentea $\mathrm{L}$. & Balsamo & Sumo das folhas para dor de ouvido \\
\hline Kalanchoe brasiliensis St. Hil. & Folha da fortuna & Chá para compressas em partes do corpo com dor \\
\hline \multicolumn{3}{|l|}{ Euphorbiaceae } \\
\hline Ricinus communis $\mathrm{L}$. & Mamona & Óleo das sementes contra vermes \\
\hline Phyllanthus niruri $\mathrm{L}$. & Quebra pedra & Chá para dissolver pedra nos rins \\
\hline \multicolumn{3}{|l|}{ Lamiaceae } \\
\hline Rosmarinus officinalis $\mathrm{L}$. & Alecrim & Chá das folhas como estimulante \\
\hline Hyptis suaveolens (L.) Poit. & Alfazema & Chá das folhas para banho contra dor de cabeça \\
\hline Plectranthus barbatus Andrews & Boldo & Chá para indigestão, dor no estômago e fígado \\
\hline Leucas martinicensis (Jacq.) R.Br. & Cordão-de-São-Francisco & Chá contra reumatismo \\
\hline Mentha piperita & Hortelã & Chá para gripe \\
\hline Plectranthus amboinicus (Lour.) Spreng. & Hortelã pimenta & Chá e lambedor para gripe e dor na garganta \\
\hline Ocimum basilicum $\mathrm{L}$. & Manjericão & Xarope para a gripe \\
\hline Mentha pulegium L. & Poejo & Lambedor para gripe \\
\hline Ocimum gratissimum $\mathrm{L}$. & Alfavaca & Xarope para a gripe \\
\hline \multicolumn{3}{|l|}{ Lauraceae } \\
\hline Cinnamomum zeylanicum Bryn. & Canela & Chá para melhorar a circulação do sangue \\
\hline \multicolumn{3}{|l|}{ Liliaceae } \\
\hline Aloe arborecens Mill. & Babosa & Gel das folhas para queimaduras \\
\hline Smilax brasiliensis Spreng. & Japecanga & Chá da raiz como depurativo \\
\hline \multicolumn{3}{|l|}{ Malvaceae } \\
\hline Gossypium barbadense $\mathrm{L}$. & Algodão & Chá das folhas usado no banho após o parto \\
\hline \multicolumn{3}{|l|}{ Moraceae } \\
\hline Brosimum gaudichaudii Trécul & Mama-cadela & Chá das folhas para vitiligo \\
\hline \multicolumn{3}{|l|}{ Nyctaginaceae } \\
\hline Mirabilis jalapa L. & Maravilha & Chá das raízes para os rins \\
\hline \multicolumn{3}{|l|}{ Plantaginaceae } \\
\hline Plantago major $\mathrm{L}$. & Tansagem & Chá para dor de garganta \\
\hline \multicolumn{3}{|l|}{ Poaceae } \\
\hline Cymbopogon citratus (DC) Stapf & Capim-cidreira & Chá calmante \\
\hline \multicolumn{3}{|l|}{ Poligonaceae } \\
\hline Polygonum bydropiper $\mathrm{L}$. & Erva-de-bicho & Chá cicatrizante \\
\hline \multicolumn{3}{|l|}{ Punicaceae } \\
\hline Punica granatum $\mathrm{L}$. & Romã & Chá da casca do fruto para a garganta e rouquidão \\
\hline \multicolumn{3}{|l|}{ Rutaceae } \\
\hline Ruta graveolens $\mathrm{L}$. & Arruda & Chá para lavar os olhos \\
\hline Pilocarpus microphyllus Stapf ex Wardleworth & Jaborandi & Chá para problemas com os rins e para os cabelos \\
\hline \multicolumn{3}{|l|}{ Solanaceae } \\
\hline Solanum paniculatum $\mathrm{L}$. & Jurubeba & Frutos no azeite para os rins e fígado \\
\hline \multicolumn{3}{|l|}{ Verbenaceae } \\
\hline Lippia alba (Mill.) N.E. Br. & Erva-cidreira & Chá calmante e digestivo \\
\hline Vitaceae & & \\
\hline Cissus verticillata (L.) Nicholson \& C.E.Jarvis & Insulina & Chá para diabetes \\
\hline Zingiberaceae & & \\
\hline Curcuma longa $\mathrm{L}$. & Açafrão & Chá para o fígado \\
\hline Alpinia zerumbet (Pers.) B.L. Burtt. \& R.M. Sm. & Colônia & Chá das folhas para pressão alta \\
\hline Zingiber officinale Roscoe. & Gengibre & Chá para gripes e resfriados \\
\hline
\end{tabular}

\section{DISCUSSÃO}

A área apresentada pelos quintais foi bem menor do que a área dos quintais em cidades do interior de Mato Grosso. Em Alta Floresta, a área média relatada foi de $703 \mathrm{~m}^{2}$ (OLIVEIRA et al., 2019), em Cáceres de 1.890,5 m² (PEREIRA; FIGUEIREDO NETO, 2015), em Mirassol do Oeste $97 \%$ dos lotes variaram entre 200 a $1.000 \mathrm{~m}^{2}$ (CARNIELLO et al., 2010), em Rosário Oeste a área média dos quintais analisados foi de $622 \mathrm{~m}^{2}$, incluindo a área do domić́lio (AMARAL; GUARIM NETO, 2008),
Com relação ao período de residência no quintal (Figura 1A) os resultados são semelhantes aos encontrados por Rondon Neto et al. (2004) em Teixeira Soares/PR, mas Pereira; Figueiredo Neto (2015) observaram maior tempo de permanência nos quintais em Cáceres (38,8 anos). Em geral, o maior tempo de permanência nos quintais favorece a diversidade de espécies.

Quanto a faixa etária (Figura 2A) o resultado difere de outros estudos em que as pessoas responsáveis pelos quintais apresentaram faixa etária maior. Siviero et al. (2012) 
encontraram forte relação entre idade do morador e a riqueza de espécies medicinais presente nos quintais urbanos de Rio Branco/AC, evidenciando que pessoas mais idosas tendem a conservar as práticas da medicina popular tradicional, cujo conhecimento relativo ao uso de plantas é, geralmente, expresso no cultivo das espécies na residência. Amaral; Guarim Neto (2008) relataram que informantes mais idosos são os que possuem maior conhecimento sobre o uso de plantas medicinais. Carniello et al. (2010) reportaram que a maioria dos informantes entrevistados (90\%) em Mirassol do Oeste/MT são aposentadas e dispõem de tempo integral para convívio no espaço da moradia e realização de atividades relacionadas, principalmente, com a administração e manejo do quintal, como o cultivo de plantas.

Por se tratar da capital do estado, a percentagem de pessoas de outras regiões é menor (Figura 2B), diferindo de estudos conduzidos em municípios do interior em que a predominância é de pessoas oriundas da região sul do Brasil refletindo o processo de colonização (GUARIM NETO; NOVAIS, 2008).

Verificou-se há um equilíbrio em relação ao gênero na execução de tarefas nos quintais (Figura 3), como também constatado por Santos et al. (2013), entretanto, difere da maioria dos estudos em que há predominância da mulher como responsável pelo quintal (FLORENTINO et al., 2007; AMARAL et al., 2016).

Uma característica marcante dos moradores dos quintais envolvidos nesta entrevista foi que todos adquirem a maioria das plantas através de doação ou troca com vizinhos e parentes. A estratégia de aquisição de mudas de plantas pelos entrevistados ocorre numa relação entre vizinhos e parentes no mesmo bairro ou de outros ou ainda com parentes que moram em outras cidades e até mesmo outros estados. Amaral; Guarim Neto (2008) verificaram em Rosário Oeste/MT que a maioria das espécies vegetais encontradas nos quintais foi adquirida por meio da doação por parentes, vizinhos e amigos.

As maior frequência das famílias Lamiaceae, Asteraceae e Euphorbiaceae (Figura 4) também foi encontrada por De David; Pasa (2015) e Moreira; Guarim Neto (2015) em estudo conduzido em Várzea Grande/MT e Tangará da Serra/MT, respectivamente, mas os estudos conduzidos em outras cidades Mato-grossenses registraram a predominância de outras famílias, como Lamiaceae, Fabaceae e Myrtaceae (OLIVEIRA et al., 2019), Arecaceae, Rutaceae, Anacardiaceae e Myrtaceae em Cáceres/MT (PEREIRA; FIGUEIREDO NETO, 2015), Solanaceae, Asteraceae, Lamiaceae, Rosaceae e Verbenaceae em Mirassol do Oeste/MT (CARNIELLO et al., 2010).

As espécies presentes em $66,7 \%$ dos quintais foram Malpighia glabra L., Mangifera indica L., Annona squamosa L. e Carica papaya L (Tabela 1). Estas frutíferas sempre estão presentes em quintais brasileiros e representam uma fonte de vitaminas e minerais. $M$. glabra é rica em vitamina $C$ e muito utilizada em sucos, $M$. indica é rica em vitamina A e potássio, A. squamosa e C. papaya são ricas em potássio e consumidas, preferencialmente, como frutas frescas.

Garcia et al. (2015) afirmam que os quintais exercem papel importante na segurança alimentar das famílias, pela riqueza de espécies encontradas nos estratos arbóreos e arbustivos, em sua maioria por espécies que proporcionam alimentação saudável, com riqueza de nutrientes. As frutas constituem valiosas fontes de nutrientes para as famílias, sendo uma alternativa econômica para o consumo destes produtos em épocas de crise.

As espécies exóticas foram mais frequentes $(60 \%)$, no entanto, espécies da flora local, como a bocaiúva (Acrocomia aculeata), a mama-cadela (Brosimum gaudichaudii), o assa-peixe (Vernonia polyanthes) e a imbaúba (Cecropia pachystachya) estavam presentes nos quintais. As espécies nativas arbóreas são obtidas de mudas provenientes de sítios e outras áreas de Cerrado próximas ao bairro e, as herbáceas ocorrem espontaneamente, nesses espaços, como quebra-pedra (Phylanthus niruri), vassourinha (Scoparia dulcis) e cipó-milhomens (Aristolochia esperanzae). O cultivo das espécies nativas é importante porque forma banco de germoplasma, que garante a variabilidade genética de muitas dessas espécies.

A predominância de espécies exóticas também foi verificada por Amaral \& Guarim Neto (2008) em Rosário Oeste/MT e Pereira \& Figueiredo Neto (2015) em estudo realizado em Cáceres/MT.

Com relação ao hábito de crescimento predominou o herbáceo $(51,2 \%)$ seguido do arbustivo $(30,4 \%)$ e do arbóreo $(18,4 \%)$. Distribuição semelhante foi observada em outros estudos (PILLA et al., 2006; ALBERTASSE et al., 2010). A maior frequência de espécies herbáceas possivelmente devese à facilidade de se cultivar ervas em quintais (PILLA et al., 2006) e também porque boa parte destas espécies ocorre espontaneamente como o chapéu-de-couro, vassourinha, erva-de-santa-maria, penicilina, picão, etc.

A categoria com maior número de espécies foi a medicinal $(38,4 \%)$ com 29 famílias, seguida da ornamental $(24,8 \%)$ e frutífera $(18,4 \%)$ (Figura 5). Em outros levantamentos realizados no Estado, também se destacou a categoria medicinal (SPILLER et al., 2016; SILVA et al., 2019), possivelmente pela tradição da medicina popular e por toda a deficiência do sistema de saúde oficial. Neste estudo também se verificou o mesmo que Silva et al. (2019) em quintais de Alta Floresta/MT, quanto à categoria de ornamentais, em que muitas espécies são plantadas com o objetivo de gerar sombra para os moradores sendo o espaço onde os adultos conversam e descansam, as crianças brincam e se realizam as festas.

As espécies medicinais são usadas para diferentes sintomas de doenças (Tabela 2) com predominância da preparação na forma de chá $(77 \%)$. As famílias mais frequentes foram Lamiaceae $(18,7 \%)$ e Asteraceae $(16,7 \%)$. Outros estudos etnobotânicos conduzidos em diferentes estados com espécies de uso medicinal também indicaram a predominância das famílias Lamiaceae e Asteraceae. Entre estes estudos citam-se Pilla et al. (2006) em São Paulo, Albertasse et al. (2010) no estado do Espírito Santo, Moreira; Guarim Neto (2015) no Mato Grosso.

A família Lamiaceae tem distribuição cosmopolita e grande importância terapêutica (PAULINO et al., 2012). As espécies desta família são ricas em óleos essenciais com propriedades aromáticas e medicinais (ABDEL-MOGIB et al., 2002), principalmente antimicrobiana e anti-inflamatória, com efeitos comprovados por estudos científicos (LORENZI; MATOS, 2008).

\section{CONCLUSÕES}

Os quintais do bairro Nossa Senhora Aparecida em Cuiabá, Mato Grosso apresentam grande diversidade vegetal. As famílias mais frequentes são Lamiaceae, Asteraceae e Euphorbiaceae, com destaque para as espécies Malpighia 
glabra L., Mangifera indica L., Annona squamosa L. e Carica papaya L. O uso medicinal da maioria das espécies indica a importância do incentivo à manutenção da diversidade nos quintais.

\section{AGRADECIMENTOS}

Ao Conselho Nacional de Desenvolvimento Científico e Tecnológico (CNPq) pela Bolsa de Produtividade concedida à segunda autora.

\section{REFERÊNCIAS}

ABDEL-MOGIB, M.; ALBAR, H. A.; BATTERJEE, S. M. Chemistry of the Genus Plectranthus. Molecules, Basel, v. 7, n. 2, p. 271-301, 2002. DOI: 10.3390/70200271

ALBERTASSE, P. D.; THOMAZ, L. D.; ANDRADE, M. A. Plantas medicinais e seus usos na comunidade da Barra do Jucu, Vila Velha, ES. Revista Brasileira de Plantas Medicinais, Botucatu, v. 12, n. 3, p. 250-260, 2010. DOI:10.1590/S1516-05722010000300002

ALBUQUERQUE, U. P.; CUNHA, L. V. F. C.; LUCENA, R. F. P.; ALVES, R. R. N. (Eds.) Methods and Techniques in Ethnobiology and Ethnoecology, New York: Springer Protocols Handbooks, 2014. 480p. DOI: https://doi.org/10.1007/978-1-4614-8636-7

ALVARES, C. A.; STAPE, J. L.; SENTELHAS, P. C.; GONÇALVES, J. L. M.; PAROVEK, G. Köppen's climate classification map for Brazil. Meteorologische Zeitschrift, Berlin, v. 22, n. 6, p. 711-728, 2014 DOI: $10.1127 / 0941-2948 / 2013 / 0507$

AMARAL C. N.; COELHO-DE-SOUZA, G. P.; SCHUCH, I.; SOUZA, M. Contribuições da produção de autoconsumo em quintais para a segurança alimentar e nutricional e renda em Jangada, Baixada Cuiabana, MT. Guaju, Matinhos, v. 2, n. 1, p. 102-119, 2016. DOI: http:/ /dx.doi.org/10.5380/guaju.v2i1.46425

AMARAL, C. N.; GUARIM NETO, G. Os quintais como espaços de conservação e cultivo de alimentos: um estudo na cidade de Rosário Oeste (Mato Grosso, Brasil). Boletim do Museu Paraense Emílio Goeldi. Ciências Humanas, Belém, v. 3, n. 3, p. 329-341, 2008. DOI: $\quad$ https://doi.org/10.1590/S198181222008000300004

AMOROZO, M. C. M.; VIERTLER, R. B. A abordagem qualitativa na coleta e análise de dados em etnobiologia e etnoecologia. In: ALBUQUERQUE, U. P.; LUCENA, R. F. P.; CUNHA, L. V. F. C. Métodos e técnicas na pesquisa etnobiológica e etnoecológica. Recife: NUPEEA, 2010. p.67-82.

BAJPAI, S.; SHARMA, A. K.; KANUNGO, V. K. Traditional home gardens: A preserve of medicinal plants. International Journal of Herbal Medicine, v. 1, n. 2, p. 152-161, 2013.

CARNIELLO, M. A.; SILVA, R. S.; CRUZ, M. A. B.; GUARIM NETO, G. Quintais urbanos de Mirassol D'Oeste-MT, Brasil: uma abordagem etnobotânica. Acta Amazônica, Manaus, v. 40, n. 3, p. 451-470, 2010. DOI: https://doi.org/10.1590/S0044-59672010000300005.

DE DAVID, M.; PASA, M. G. As plantas medicinais e a etnobotânica em Várzea Grande, MT, Brasil. Interações, Campo Grande, v. 16, n. 1, p. 97-108, 2015. DOI: https://doi.org/10.1590/1518-70122015108.

FLORENTINO, A. T. N.; ARAUJO, E. L.; ALBUQUERQUE, U. P. Contribuição de quintais agroflorestais na conservação de plantas da Caatinga, município de Caruaru, PE, Brasil. Acta Botânica Brasílica, São Paulo, v. 21, n. 1 p. 37-47, 2007. DOI: https://doi.org/10.1590/S0102-33062007000100005

GAO, J.; HE, T.; LI, Q. M. Traditional home-garden conserving genetic diversity: a case study of Acacia pennata in southwest China. Conservation Genetics, v. 13, n. 4, p. 891-898, 2012. DOI: 10.1007 / s10592-012-0338-x

GARCIA, B. N. R.; VIEIRA, T. A.; OLIVEIRA, F. A. Quintais agroflorestais e segurança alimentar em uma comunidade rural na Amazônia Oriental. Revista da Faculdade de Agronomia de La Plata, Maracaibo, v. 114, n. 1, p. 67-73, 2015.

GUARIM NETO, G.; AMARAL, C. N. Aspectos etnobotânicos de quintais tradicionais dos moradores de Rosário Oeste, Mato Grosso, Brasil. Polibotánica, Cidade do México, v. 29, p. 191-212, 2010.

GUARIM NETO, G.; NOVAIS, A. M. Composição florística dos quintais da cidade de Castanheira. In: GUARIM NETO, G.; CARNIELlO, M. A. (Orgs.). Quintais mato-grossenses: espaços de conservação e reprodução de saberes. Cáceres: EDUNEMAT, 2008. 203p.

IBGE_Instituto Brasileiro de Geografia e Estatística. Censo Demográfico 2019. Disponível em: < https://cidades.ibge.gov.br/brasil/mt/cuiaba/panoram a>.

KUMAR, B. M.; NAIR, P. K. R. Tropical home gardens: A time tested example of sustainable agroforestry. Netherlands: Springer Science, 2006. 380p.

LORENZI, H.; MATOS, F. J. A. M. Plantas Medicinais no Brasil: nativas e exóticas. 2 ed. Nova Odessa: Instituto Plantarum, 2008. 544p.

MOREIRA, R. P. M.; GUARIM NETO, G. A flora medicinal dos quintais de Tangará da Serra, Mato Grosso, Brasil. Biodiversidade, Cuiabá, v. 14, n. 1, p. 63-83, 2015.

OLIVEIRA, W. A. Os recursos vegetais e o saber local nos quintais da comunidade de Santo Antônio do Caramujo, Cáceres, Mato Grosso, Brasil. 104f. Dissertação (Mestrado em Ciências Florestais e Ambientais) - Universidade Federal de Mato Grosso, Cuiabá. 2013.

PAULINO, R. C.; HENRIQUES, G. P. S. A.; MOURA, O. N. S.; COElHO, M. F. B.; AZEVEDO, R. A. B. Medicinal plants at the Sítio do Gois, Apodi, Rio Grande do Norte State, Brazil. Revista Brasileira de Farmacognosia, Curitiba, v. 22, n. 1, p. 29-39, 2012. DOI: https://doi.org/10.1590/S0102695X2011005000203

PEREIRA, P. V. M.; FIGUEIREDO NETO, L. F. Conservação de espécies florestais: um estudo em quintais agroflorestais no município de Cáceres - MT. Revista Eletrônica em Gestão, Educação e Tecnologia Ambiental, Santa Maria, v. 19 n. 3, p. 783793, 2015.

PILlA, M. A. C.; AMOROZO, M. C. M.; FURLAN, A. Obtenção e uso das plantas medicinais no distrito de Martim Francisco, Município de Mogi-Mirim, SP, Brasil. Acta Botânica Brasílica, Brasília, v. 20, n. 4, p. 789-802, 2006. DOI: https://doi.org/10.1590/S010233062006000400005

RONDON NETO, R. M.; BYCZKOVSKI, A.; WINNICKI, J. A.; SIMÃO, S. M. M.; PASQUALOTTO, 
T. C. Os quintais agroflorestais do assentamento rural Rio da Areia, município de Teixeira Soares, PR. Cerne, Lavras, v. 10, n. 1, p. 125-135, 2004.

SANTOS, A. S.; OLIVEIRA, L. C. L.; CURADO, F. F.; AMORIM, L. O. Caracterização e desenvolvimento de quintais produtivos agroecológicos na comunidade Mem de Sá, Itaporanga d'Ajuda-Sergipe. Revista Brasileira de Agroecologia, Porto Alegre, v. 8, n. 2, p. 100-111, 2013.

SILVA, J. R. O., SILVA, I. C. O., COELHO, M. F. B., CAMILI, E. C. As plantas e seus usos nos quintais de Alta Floresta, Mato Grosso. Revista Verde de Agroecologia e Desenvolvimento Sustentável, Pombal, v. 14, n. 3, p. 420-428, 2019. DOI: https://doi.org/10.18378/rvads.v14i3.6086

SIVIERO, A.; DELUNARDO, T. A.; HAVERROTH, M.; OLIVEIRA, L. C.; MENDONÇA, A. M. S. Plantas medicinais em quintais urbanos de Rio Branco, Acre. Revista Brasileira de Plantas Medicinais, Botucatu, v. 14, n. 4, p. 598-610, 2012. DOI: https://doi.org/10.1590/S1516-05722012000400005

SPILLER, C.; COELHO, M. F. B.; GONÇALVES, V. D.; PITON, L. P.; CAMILI, E. C. Estudo etnobotânico em quintais agroflorestais em bairro na Cidade de Cuiabá, Mato Grosso. Revista Verde de Agroecologia e Desenvolvimento Sustentável, Pombal, v. 11, n. 5, p. 138-142, 2016.2 DOI: https://doi.org/10.18378/rvads.v11i5.3832

TOURINHO, H. L. Z., SILVA, M. G. C. A. Quintais urbanos: funções e papéis na casa brasileira e amazônica. Boletim do Museu Paraense Emílio Goeldi. Ciências Humanas, Belém, v. 11, n. 3, p. 633651, 2016.2 DOI: https://doi.org/10.1590/1981.81222016000300006

VIEIRA, T. A.; ROSA, L. S.; SANTOS, M. M. L. S. Agrobiodiversidade de quintais agroflorestais no município de Bonito, Estado do Pará. Revista de Ciências Agrarias, Belém, v. 55, n. 3, p. 159-166, 2012. 